\title{
Access to an Excited State via the Thermal Ring-opening of a Cyclopropylidene.
}

\author{
Matthew E. Cremeens ${ }^{\dagger}$ and Barry K. Carpenter ${ }^{*}$
}

Supporting Information.

Singlet 4-methylene-bicyclo[3.1.0]hex-2-ene-6-ylidene (1) B3LYP/6-31G*

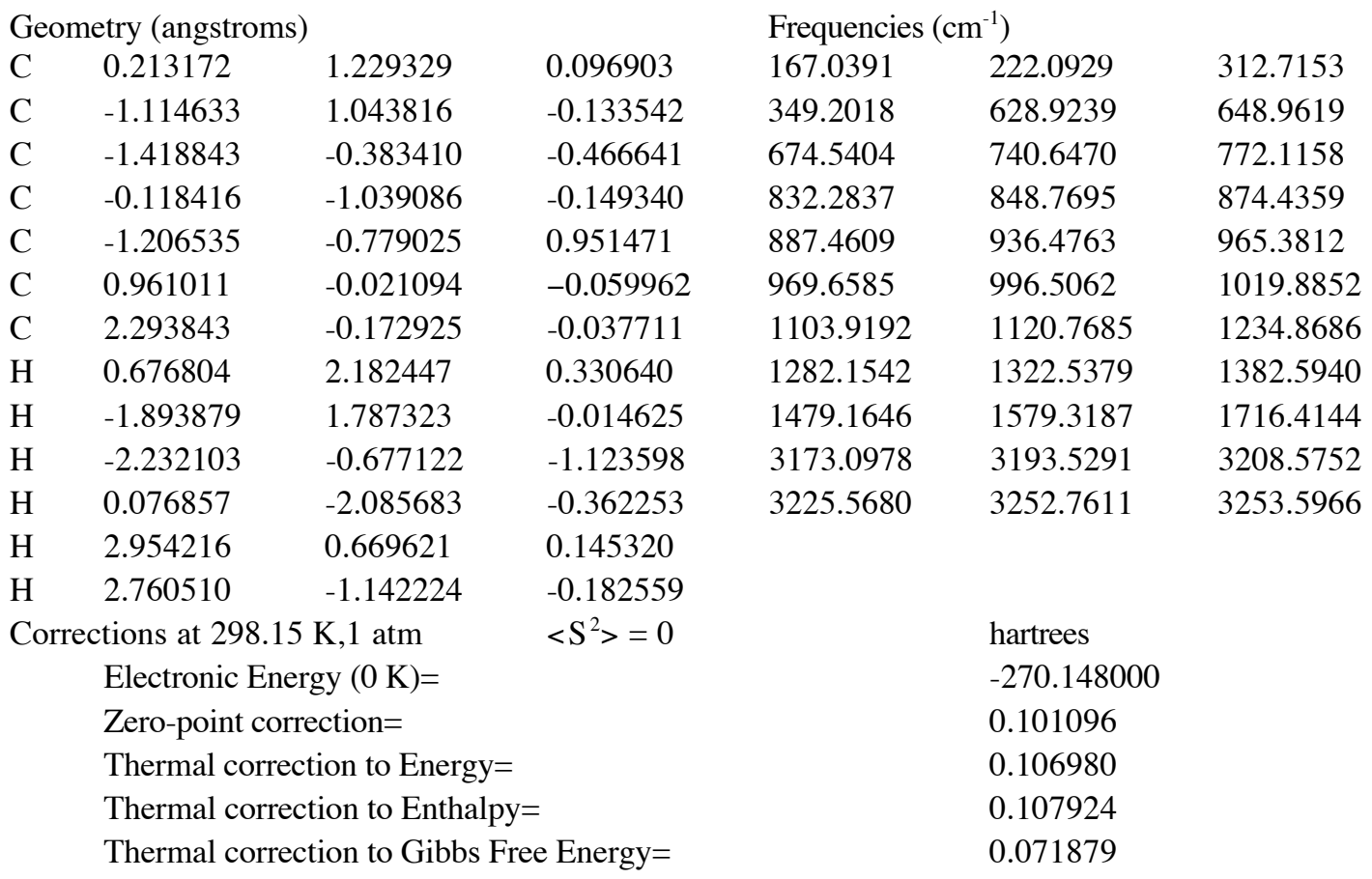


Triplet Cyclopropylidene (1) UB3LYP/6-31G*

\begin{tabular}{|c|c|c|c|c|c|c|}
\hline \multicolumn{4}{|c|}{ Geometry (angstroms) } & \multicolumn{3}{|c|}{ Frequencies $\left(\mathrm{cm}^{-1}\right)$} \\
\hline $\mathrm{C}$ & 0.260740 & 1.284632 & 0.108605 & 167.0684 & 333.2619 & 376.7417 \\
\hline $\mathrm{C}$ & -1.075061 & 1.190575 & -0.048590 & 406.7035 & 578.0910 & 621.7098 \\
\hline $\mathrm{C}$ & -1.526660 & -0.214924 & -0.261936 & 690.5960 & 697.1297 & 762.6465 \\
\hline $\mathrm{C}$ & -0.114435 & -1.061834 & -0.224832 & 813.4900 & 839.5943 & 901.6309 \\
\hline $\mathrm{C}$ & -1.173905 & -1.196693 & 0.756634 & 904.8856 & 951.2419 & 955.7974 \\
\hline $\mathrm{C}$ & 0.945735 & -0.009358 & -0.029531 & 970.3419 & 1000.0878 & 1030.3067 \\
\hline $\mathrm{C}$ & 2.269455 & -0.218934 & -0.012040 & 1126.4945 & 1186.9423 & 1256.6201 \\
\hline $\mathrm{H}$ & 0.801645 & 2.206060 & 0.303142 & 1281.4813 & 1339.2433 & 1375.0791 \\
\hline $\mathrm{H}$ & -1.768140 & 2.025398 & -0.015544 & 1473.5556 & 1621.8396 & 1714.6282 \\
\hline $\mathrm{H}$ & -2.264675 & -0.430153 & -1.038156 & 3112.9004 & 3117.6371 & 3166.9857 \\
\hline $\mathrm{H}$ & 0.052919 & -1.829519 & -0.983856 & 3205.1124 & 3227.1693 & 3246.7266 \\
\hline $\mathrm{H}$ & 2.968580 & 0.596435 & 0.153303 & & & \\
\hline $\mathrm{H}$ & 2.694461 & -1.209001 & -0.148754 & & & \\
\hline \multicolumn{3}{|c|}{ Corrections at $298.15 \mathrm{~K}, 1 \mathrm{~atm}$} & \multicolumn{2}{|c|}{$<\mathrm{S}^{2}>=2.004788$} & hartrees & \\
\hline & \multicolumn{4}{|c|}{ Electronic Energy $(0 \mathrm{~K})=$} & -270.125471 & \\
\hline & \multicolumn{4}{|c|}{ Zero-point correction= } & 0.101273 & \\
\hline & \multicolumn{4}{|c|}{ Thermal correction to Energy= } & 0.106918 & \\
\hline & \multicolumn{4}{|c|}{ Thermal correction to Enthalpy= } & 0.107863 & \\
\hline & \multicolumn{4}{|c|}{ Thermal correction to Gibbs Free Energy= } & 0.071234 & \\
\hline
\end{tabular}

Singlet Cyclopropylidene (1) CASSCF $(8,8) / 6-31 \mathrm{G}^{*}$

\begin{tabular}{|c|c|c|c|}
\hline \multicolumn{3}{|c|}{ Geometry (angstroms) } & endpoint of IRC \\
\hline $\mathrm{C}$ & -0.181928 & -0.972731 & -0.342909 \\
\hline $\mathrm{C}$ & -1.264391 & -1.070794 & 0.706743 \\
\hline $\mathrm{C}$ & -1.431444 & -0.114694 & -0.427426 \\
\hline $\mathrm{C}$ & -0.955250 & 1.250903 & -0.011565 \\
\hline $\mathrm{C}$ & 0.371251 & 1.264111 & 0.220289 \\
\hline $\mathrm{C}$ & 0.968896 & -0.058598 & -0.033996 \\
\hline $\mathrm{C}$ & 2.269333 & -0.385944 & -0.000594 \\
\hline $\mathrm{H}$ & -0.020005 & -1.870791 & -0.915325 \\
\hline $\mathrm{H}$ & -2.250516 & -0.226056 & -1.117074 \\
\hline $\mathrm{H}$ & -1.630546 & 2.071626 & 0.134067 \\
\hline $\mathrm{H}$ & 0.938089 & 2.114462 & 0.548910 \\
\hline $\mathrm{H}$ & 3.020548 & 0.342544 & 0.246256 \\
\hline $\mathrm{H}$ & 2.606732 & -1.384024 & -0.212295 \\
\hline
\end{tabular}

Electronic Energy $(0 \mathrm{~K})=$ -268.466375 hartrees 
Cyclopropylidene (1) T.S. B3LYP/6-31G*

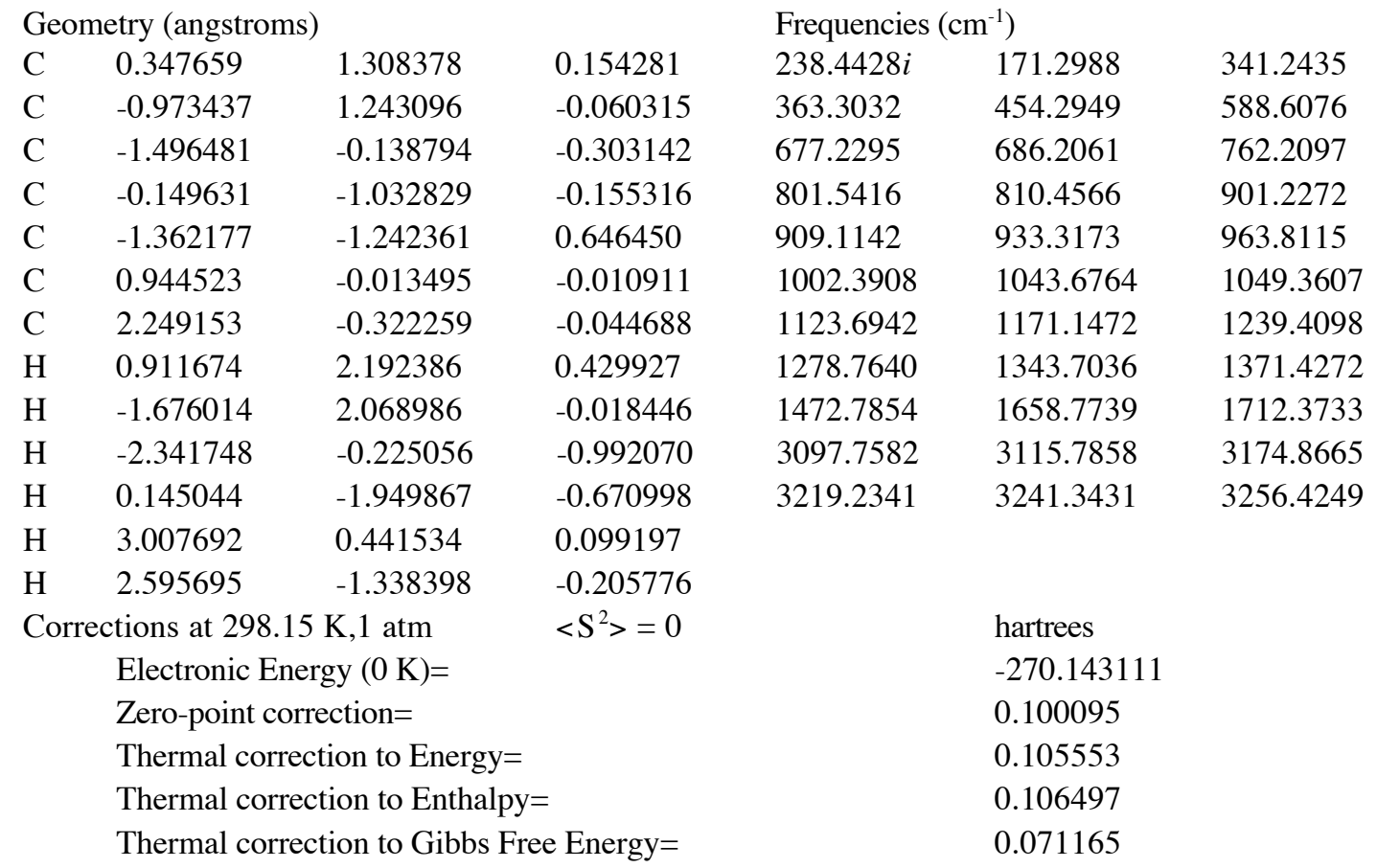

Cyclopropylidene (1) T.S. CASSCF(8,8)/6-31G*

\begin{tabular}{|c|c|c|c|c|c|c|}
\hline \multicolumn{4}{|c|}{ Geometry (angstroms) } & \multicolumn{3}{|c|}{ Frequencies $\left(\mathrm{cm}^{-1}\right)$} \\
\hline $\mathrm{C}$ & 1.342641 & 0.258753 & 0.047381 & $405.83 i$ & 159.89 & 365.94 \\
\hline $\mathrm{C}$ & 1.130405 & -1.521801 & -0.046884 & 383.03 & 446.15 & 586.52 \\
\hline $\mathrm{C}$ & -0.342529 & -1.578292 & -0.255862 & 681.24 & 706.61 & 779.83 \\
\hline $\mathrm{C}$ & -0.986052 & -0.403695 & -0.304852 & 797.89 & 855.02 & 886.93 \\
\hline $\mathrm{C}$ & -0.043030 & 0.710103 & -0.268184 & 923.42 & 960.38 & 1024.50 \\
\hline $\mathrm{C}$ & 1.817933 & -0.755535 & 0.952996 & 1039.20 & 1062.38 & 1209.54 \\
\hline $\mathrm{C}$ & -0.336282 & 2.002118 & -0.501311 & 1235.89 & 1262.49 & 1386.65 \\
\hline $\mathrm{H}$ & 2.125952 & 0.918420 & -0.295194 & 1410.09 & 1476.11 & 1525.18 \\
\hline $\mathrm{H}$ & 1.713279 & -2.308094 & -0.504455 & 1587.11 & 1728.48 & 1779.28 \\
\hline $\mathrm{H}$ & -0.810640 & -2.544188 & -0.293146 & 3313.55 & 3319.63 & 3336.97 \\
\hline $\mathrm{H}$ & -2.049728 & -0.274263 & -0.365651 & 3375.00 & 3396.86 & 3425.70 \\
\hline $\mathrm{H}$ & -1.345401 & 2.313608 & -0.701213 & & & \\
\hline $\mathrm{H}$ & 0.418561 & 2.766816 & -0.489174 & & & \\
\hline \multicolumn{5}{|c|}{ Corrections at $298.15 \mathrm{~K}, 1 \mathrm{~atm}$} & \multicolumn{2}{|c|}{ hartrees } \\
\hline & \multicolumn{4}{|c|}{ Electronic Energy $(0 \mathrm{~K})=$} & \multicolumn{2}{|c|}{-268.454849} \\
\hline & \multicolumn{4}{|c|}{ Zero-point correction= } & \multicolumn{2}{|l|}{0.105770} \\
\hline & \multicolumn{4}{|c|}{ Thermal correction to Energy= } & \multicolumn{2}{|l|}{0.111109} \\
\hline & \multicolumn{4}{|c|}{ Thermal correction to Enthalpy= } & \multicolumn{2}{|l|}{0.112054} \\
\hline & \multicolumn{4}{|c|}{ Thermal correction to Gibbs Free Energy= } & \multicolumn{2}{|l|}{0.076847} \\
\hline
\end{tabular}



Singlet $\alpha, 3$-didehydrotoluene biradical UB3LYP/6-31G*

\begin{tabular}{|c|c|c|c|c|c|c|}
\hline \multicolumn{4}{|c|}{ Geometry (angstroms) } & \multicolumn{3}{|c|}{ Frequencies $\left(\mathrm{cm}^{-1}\right)$} \\
\hline $\mathrm{C}$ & 1.173714 & 0.149067 & 0.000000 & 197.3799 & 359.3602 & 377.0631 \\
\hline $\mathrm{C}$ & 1.101145 & -1.235363 & 0.000000 & 430.2563 & 504.4870 & 522.0605 \\
\hline $\mathrm{C}$ & -0.148514 & -1.891910 & 0.000000 & 619.0749 & 651.9456 & 712.1039 \\
\hline $\mathrm{C}$ & -1.266168 & -1.075280 & 0.000000 & 742.5098 & 825.8679 & 835.6758 \\
\hline $\mathrm{C}$ & -1.269590 & 0.291223 & 0.000000 & 864.1516 & 962.7234 & 971.3656 \\
\hline $\mathrm{C}$ & 0.000000 & 0.966460 & 0.000000 & 981.6340 & 1055.8975 & 1109.1595 \\
\hline $\mathrm{C}$ & 0.079310 & 2.367551 & 0.000000 & 1180.5001 & 1259.3956 & 1323.8421 \\
\hline $\mathrm{H}$ & 2.144122 & 0.638699 & 0.000000 & 1329.0985 & 1451.3793 & 1471.9739 \\
\hline $\mathrm{H}$ & 2.015292 & -1.824732 & 0.000000 & 1515.0357 & 1525.2774 & 1601.7341 \\
\hline $\mathrm{H}$ & -0.214291 & -2.976009 & 0.000000 & 3174.4150 & 3178.5887 & 3185.6761 \\
\hline $\mathrm{H}$ & -2.187591 & 0.874160 & 0.000000 & 3196.7791 & 3207.2753 & 3267.5831 \\
\hline $\mathrm{H}$ & -0.815158 & 2.981687 & 0.000000 & & & \\
\hline $\mathrm{H}$ & 1.038248 & 2.875708 & 0.000000 & & & \\
\hline \multicolumn{3}{|c|}{ Corrections at $298.15 \mathrm{~K}, 1 \mathrm{~atm}$} & \multicolumn{2}{|c|}{$<S^{2}>=1.056094$} & hartrees & \\
\hline & \multicolumn{4}{|c|}{ Electronic Energy $(0 \mathrm{~K})=$} & -270.229068 & \\
\hline & \multicolumn{4}{|c|}{ Zero-point correction= } & 0.101586 & \\
\hline & \multicolumn{4}{|c|}{ Thermal correction to Energy= } & 0.107319 & \\
\hline & \multicolumn{4}{|c|}{ Thermal correction to Enthalpy= } & 0.108263 & \\
\hline & \multicolumn{4}{|c|}{ Thermal correction to Gibbs Free Energy= } & 0.072572 & \\
\hline
\end{tabular}

Singlet $\alpha, 3$-didehydrotoluene biradical $\operatorname{CASSCF}(8,8) / 6-31 \mathrm{G}^{*}$

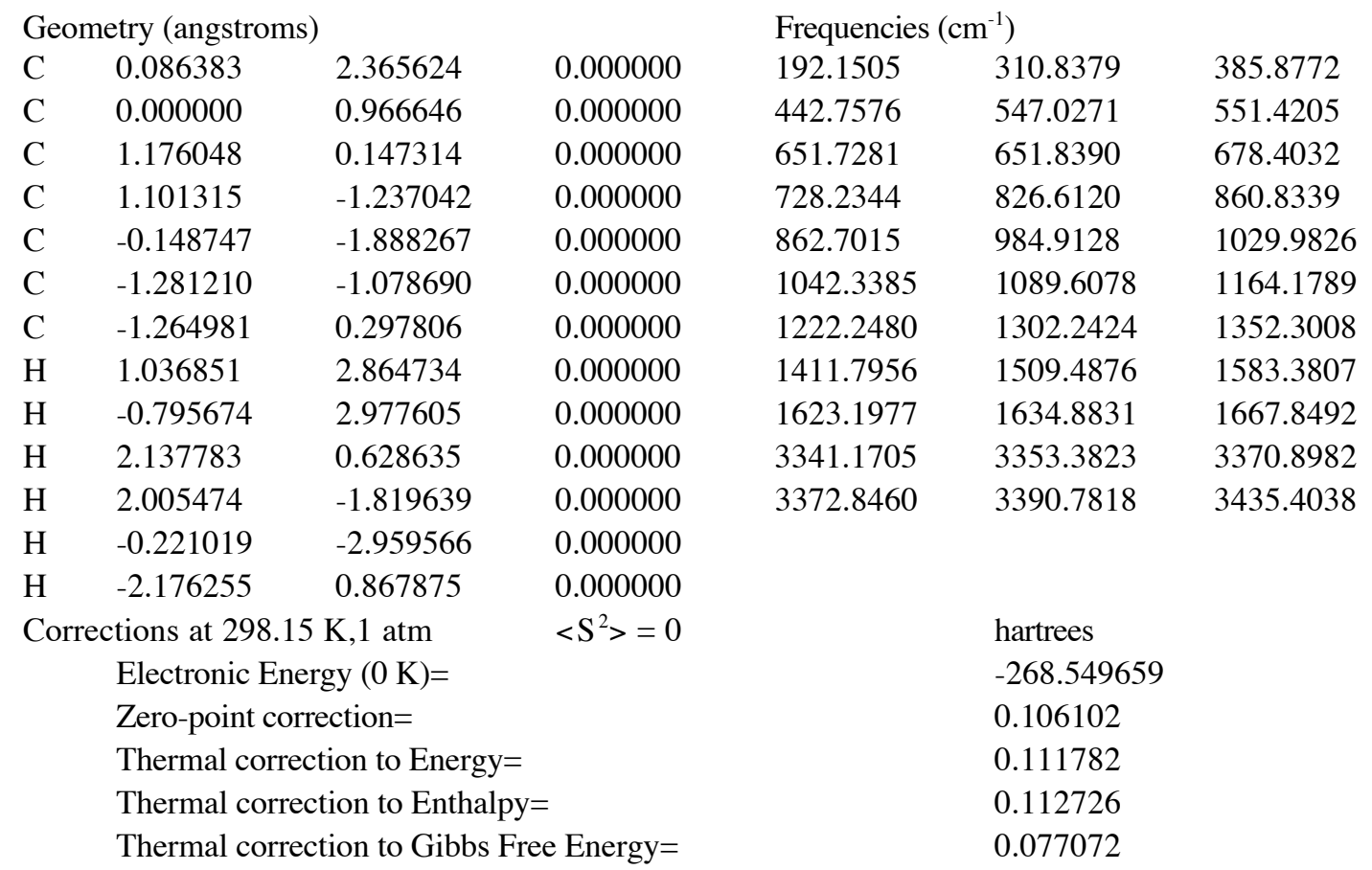



Benzannelated 2,3,5-cyclohexatrienylideneamine (7) RB3LYP/6-31G*

\begin{tabular}{lllll}
\multicolumn{2}{l}{ Geometry (angstroms) } & & & \\
$\mathrm{C}$ & -2.268732 & -1.161333 & 0.047838 & 0 \\
$\mathrm{C}$ & -2.713241 & 0.162885 & 0.091647 & \\
$\mathrm{C}$ & -1.790089 & 1.206404 & 0.060016 & \\
$\mathrm{C}$ & -0.416910 & 0.935936 & 0.012558 & \\
$\mathrm{C}$ & 0.039316 & -0.412441 & -0.030768 & \\
$\mathrm{C}$ & 0.613862 & 1.958865 & -0.266730 & \\
$\mathrm{C}$ & -0.905011 & -1.443487 & -0.029479 & \\
$\mathrm{C}$ & 1.512207 & -0.754105 & -0.074922 & \\
$\mathrm{C}$ & 2.401564 & 0.395144 & 0.298586 & \\
$\mathrm{~N}$ & 1.865357 & -1.983478 & -0.174270 & \\
$\mathrm{C}$ & 1.836577 & 1.577741 & 0.090740 & \\
$\mathrm{H}$ & -2.986400 & -1.976794 & 0.072855 & \\
$\mathrm{H}$ & -3.776279 & 0.381146 & 0.147345 & \\
$\mathrm{H}$ & -2.127634 & 2.239553 & 0.067962 & \\
$\mathrm{H}$ & -0.546751 & -2.466677 & -0.078725 & \\
$\mathrm{H}$ & 3.239599 & 0.202128 & 0.965025 & \\
$\mathrm{H}$ & 0.396083 & 2.773797 & -0.953571 & \\
$\mathrm{H}$ & 2.886616 & -2.062461 & -0.197924 &
\end{tabular}

Electronic Energy $(0 \mathrm{~K})=$ -439.913630 hartrees

Benzannelated 2,3,5-cyclohexatrienylideneamine (8) RB3LYP/6-31G*

\begin{tabular}{lllll}
\multicolumn{3}{l}{ Geometry (angstroms) } & & \\
$\mathrm{C}$ & -2.283475 & 1.134243 & 0.074453 & 0 \\
$\mathrm{C}$ & -2.710195 & -0.194002 & 0.084104 & \\
$\mathrm{C}$ & -1.769803 & -1.222077 & 0.031676 & \\
$\mathrm{C}$ & -0.401054 & -0.935212 & -0.004188 & \\
$\mathrm{C}$ & 0.043854 & 0.420802 & -0.026158 & \\
$\mathrm{C}$ & 0.640429 & -1.951324 & -0.277708 & \\
$\mathrm{C}$ & -0.921282 & 1.431180 & 0.005010 & \\
$\mathrm{C}$ & 1.529142 & 0.765622 & -0.072335 & \\
$\mathrm{C}$ & 2.393736 & -0.378950 & 0.354451 & \\
$\mathrm{~N}$ & 2.039201 & 1.929284 & -0.229659 & \\
$\mathrm{C}$ & 1.853007 & -1.562567 & 0.100295 & \\
$\mathrm{H}$ & -3.008256 & 1.942008 & 0.122740 & \\
$\mathrm{H}$ & -3.769901 & -0.428305 & 0.134349 & \\
$\mathrm{H}$ & -2.092363 & -2.259957 & 0.018732 & \\
$\mathrm{H}$ & -0.616098 & 2.474896 & -0.007409 & \\
$\mathrm{H}$ & 3.213258 & -0.173160 & 1.036649 & \\
$\mathrm{H}$ & 0.436003 & -2.757086 & -0.979458 & \\
$\mathrm{H}$ & 1.316801 & 2.650322 & -0.335597 &
\end{tabular}

Electronic Energy $(0 \mathrm{~K})=$ -439.909597 hartrees 
Data for Figure 1

$\operatorname{SA}\left(50: 50 / \mathrm{S}_{0}: \mathrm{S}_{1}\right) \operatorname{CASSCF}(8,8) / 6-31 \mathrm{G}^{*}$

$\begin{array}{lll}\text { RXN Coord. (amu }{ }^{1 / 2} \text { bohr) } & \mathrm{S}_{0} \text { (hartrees) } & \mathrm{S}_{1} \text { (hartrees) } \\ 0 & -268.441525 & -268.337268 \\ 0.29997 & -268.442824 & -268.335070 \\ 0.59989 & -268.444266 & -268.353825 \\ 0.89982 & -268.446058 & -268.372007 \\ 1.19974 & -268.448232 & -268.389245 \\ 1.49962 & -268.450765 & -268.405367 \\ 1.79938 & -268.453694 & -268.420154 \\ 2.09806 & -268.457351 & -268.432844 \\ 2.39326 & -268.462950 & -268.441330 \\ 2.69119 & -268.471266 & -268.445380 \\ 2.99033 & -268.480929 & -268.447848 \\ 3.58957 & -268.499759 & -268.450476 \\ 4.18889 & -268.515349 & -268.451563 \\ 4.78813 & -268.527229 & -268.452312 \\ 5.38713 & -268.535559 & -268.453345 \\ 5.98545 & -268.540431 & -268.454690 \\ 6.57663 & -268.542012 & -268.455824 \\ 7.16705 & -268.541676 & -268.456207 \\ 7.76286 & -268.541458 & -268.456260\end{array}$

Data for Figure 2

\begin{tabular}{lllll} 
Fraction & Fraction & \multicolumn{2}{l}{$\mathrm{SA}\left(50: 50 / \mathrm{S}_{0}: \mathrm{S}_{1}\right) \mathrm{CASSCF}(8,8) / 6-31 \mathrm{G}^{*}$} & CASSCF $(8,8) / 6-31 \mathrm{G}^{*}$ \\
of C.I. & of T.S. & $\mathrm{S}_{0}$ (hartrees) & $\mathrm{S}_{1}$ (hartrees) & E (hartrees) \\
0.00 & 1.00 & -268.441525 & -268.337268 & -268.454849 \\
0.05 & 0.95 & -268.441767 & -268.333520 & -268.454645 \\
0.10 & 0.90 & -268.441963 & -268.335929 & -268.454094 \\
0.15 & 0.85 & -268.441470 & -268.345045 & -268.453301 \\
0.20 & 0.80 & -268.440834 & -268.353604 & -268.452383 \\
0.25 & 0.75 & -268.440178 & -268.361681 & -268.451464 \\
0.30 & 0.70 & -268.439626 & -268.369380 & -268.450669 \\
0.35 & 0.65 & -268.439299 & -268.376808 & -268.450118 \\
0.40 & 0.60 & -268.439303 & -268.384070 & -268.449919 \\
0.45 & 0.55 & -268.439730 & -268.391261 & -268.450163 \\
0.50 & 0.50 & -268.440639 & -268.398457 & -268.450910 \\
0.55 & 0.45 & -268.442063 & -268.405705 & -268.452192 \\
0.60 & 0.40 & -268.443997 & -268.413021 & -268.454002 \\
0.65 & 0.35 & -268.446395 & -268.420386 & -268.456293 \\
0.70 & 0.30 & -268.449171 & -268.427746 & -268.458978 \\
0.75 & 0.25 & -268.452205 & -268.435013 & -268.461934 \\
0.80 & 0.20 & -268.455341 & -268.442068 & -268.465004 \\
0.85 & 0.15 & -268.458400 & -268.448770 & -268.468008 \\
0.90 & 0.10 & -268.461186 & -268.454961 & -268.470749 \\
0.95 & 0.05 & -268.463497 & -268.460480 & -268.473023
\end{tabular}


1.00

0.00 
Data for Figure 2 (continued)

\begin{tabular}{lllll} 
Fraction & Fraction & \multicolumn{4}{l}{ TDUB3LYP/6-31G* } & \\
of C.I. & of T.S. & $\mathrm{S}_{0}$ (hartrees) & $<\mathrm{S}^{2}>$ & $\mathrm{S}_{1}(\mathrm{eV})$ \\
0.00 & 1.00 & -270.143313 & 0.00 & 2.5394 \\
0.05 & 0.95 & -270.143749 & 0.00 & 2.5407 \\
0.10 & 0.90 & -270.143846 & 0.00 & 2.3948 \\
0.15 & 0.85 & -270.143693 & 0.00 & 2.2400 \\
0.20 & 0.80 & -270.143391 & 0.00 & 2.0819 \\
0.25 & 0.75 & -270.143046 & 0.00 & 1.9234 \\
0.30 & 0.70 & -270.142767 & 0.00 & 1.7665 \\
0.35 & 0.65 & -270.142661 & 0.00 & 1.6129 \\
0.40 & 0.60 & -270.142824 & 0.00 & 1.4638 \\
0.45 & 0.55 & -270.143338 & 0.00 & 1.3203 \\
0.50 & 0.50 & -270.144264 & 0.00 & 1.1830 \\
0.55 & 0.45 & -270.145631 & 0.00 & 1.0522 \\
0.60 & 0.40 & -270.147440 & 0.00 & 0.9281 \\
0.65 & 0.35 & -270.149653 & 0.00 & 0.8108 \\
0.70 & 0.30 & -270.152199 & 0.00 & 0.7002 \\
0.75 & 0.25 & -270.154971 & 0.00 & 0.5963 \\
0.80 & 0.20 & -270.157832 & 0.00 & 0.4987 \\
0.85 & 0.15 & -270.160625 & 0.0046 & 0.4092 \\
0.90 & 0.10 & -270.163198 & 0.0441 & 0.3432 \\
0.95 & 0.05 & -270.165381 & 0.0786 & 0.2931 \\
1.00 & 0.00 & -270.166995 & 0.1087 & 0.1849
\end{tabular}

Conical Intersection $\operatorname{SA}\left(50: 50 / \mathrm{S}_{0}: \mathrm{S}_{1}\right) \operatorname{CASSCF}(8,8) / 6-31 \mathrm{G}^{*}$

$\begin{array}{lllll}\text { Geometry (angstroms) } & & & \\ \mathrm{C} & -0.118488 & -1.214220 & 0.021540 & \mathrm{~S}_{0} \\ \mathrm{C} & 1.269313 & -1.423858 & -0.206940 & \mathrm{~S}_{1} \\ \mathrm{C} & 1.731318 & -0.095114 & 0.085962 & \\ \mathrm{C} & 1.072621 & 1.218474 & 0.044383 & \\ \mathrm{C} & -0.263093 & 1.291057 & -0.087473 & \\ \mathrm{C} & -0.947301 & 0.005747 & -0.005767 & \\ \mathrm{C} & -2.288074 & -0.099180 & 0.041880 & \\ \mathrm{H} & -0.693495 & -2.087437 & 0.304575 & \\ \mathrm{H} & 2.769067 & -0.022498 & 0.386677 & \\ \mathrm{H} & 1.694852 & 2.095634 & 0.054372 & \\ \mathrm{H} & -0.809584 & 2.206008 & -0.221829 & \\ \mathrm{H} & -2.772871 & -1.053510 & 0.138330 & \\ \mathrm{H} & -2.925749 & 0.764367 & -0.023634 & \end{array}$

Electronic Energy $(0 \mathrm{~K})=$ -268.465167 hartrees -268.465136 hartrees 
The geometries (in angstroms and degrees) that were used to generate the LST.

\begin{tabular}{|c|c|c|c|c|c|c|c|c|c|c|}
\hline & C.I. & T.S. & $\mathbf{Z m}$ & & & & & & & \\
\hline r1 & 1.4220 & 1.4404 & 1 & $\mathrm{C}$ & & & & & & \\
\hline r2 & 1.4369 & 1.4351 & 2 & $\mathrm{C}$ & 1 & r1 & & & & \\
\hline r3 & 1.4701 & 1.4888 & 3 & $\mathrm{C}$ & 2 & r2 & 1 & $\mathrm{a} 2$ & & \\
\hline r4 & 1.3442 & 1.3402 & 4 & $\mathrm{C}$ & 3 & r3 & 2 & a3 & 1 & $\mathrm{~d} 3$ \\
\hline $\mathrm{r} 5$ & 1.4584 & 1.4599 & 5 & $\mathrm{C}$ & 4 & $\mathrm{r} 4$ & 3 & a4 & 2 & $\mathrm{~d} 4$ \\
\hline r6 & 1.3457 & 1.3452 & 6 & $\mathrm{C}$ & 5 & r5 & 4 & a5 & 3 & $\mathrm{~d} 5$ \\
\hline r7 & 1.0832 & 1.0799 & 7 & $\mathrm{C}$ & 6 & r6 & 5 & a6 & 4 & d6 \\
\hline r8 & 1.0829 & 1.0804 & 8 & $\mathrm{H}$ & 1 & $\mathrm{r} 7$ & 2 & a7 & 3 & d7 \\
\hline r9 & 1.0755 & 1.0740 & 9 & $\mathrm{H}$ & 3 & r8 & 4 & a8 & 5 & $\mathrm{~d} 8$ \\
\hline r10 & 1.0742 & 1.0732 & 10 & $\mathrm{H}$ & 4 & $\mathrm{r} 9$ & 5 & a9 & 6 & $\mathrm{~d} 9$ \\
\hline r11 & 1.0755 & 1.0749 & 11 & $\mathrm{H}$ & 5 & r10 & 6 & a10 & 7 & d10 \\
\hline r12 & 1.0747 & 1.0746 & 12 & $\mathrm{H}$ & 7 & r11 & 6 & a11 & 5 & d11 \\
\hline $\mathrm{a} 2$ & 98.320 & 77.283 & 13 & $\mathrm{H}$ & 7 & r12 & 6 & a12 & 5 & $\mathrm{~d} 12$ \\
\hline a3 & 132.566 & 126.302 & & & & & & & & \\
\hline $\mathrm{a} 4$ & 119.754 & 116.547 & & & & & & & & \\
\hline a5 & 114.401 & 110.951 & & & & & & & & \\
\hline a6 & 122.557 & 125.987 & & & & & & & & \\
\hline a7 & 116.182 & 112.971 & & & & & & & & \\
\hline a8 & 112.168 & 116.531 & & & & & & & & \\
\hline a9 & 122.126 & 125.286 & & & & & & & & \\
\hline a10 & 121.266 & 123.341 & & & & & & & & \\
\hline a11 & 121.735 & 121.013 & & & & & & & & \\
\hline a12 & 121.456 & 121.897 & & & & & & & & \\
\hline $\mathrm{d} 3$ & -24.645 & -70.867 & & & & & & & & \\
\hline d4 & 12.071 & 48.570 & & & & & & & & \\
\hline d5 & 7.469 & 5.630 & & & & & & & & \\
\hline d6 & 171.979 & 169.352 & & & & & & & & \\
\hline d7 & -154.140 & -126.845 & & & & & & & & \\
\hline d8 & -168.793 & -148.405 & & & & & & & & \\
\hline d9 & -176.801 & -178.421 & & & & & & & & \\
\hline d10 & -6.817 & -10.503 & & & & & & & & \\
\hline d11 & 2.854 & 2.588 & & & & & & & & \\
\hline d12 & -177.720 & -177.900 & & & & & & & & \\
\hline
\end{tabular}

